()

CORPUS PUBLISHERS

\section{Current Research} in Emergency Medicine (CREM)

ISSN: 2832-5699

Volume 1, Issue 1, 2021

Article Information

Received date : 22 September, 2021

Published date: 30 September, 2021

*Corresponding author

Jacky Sia, Emergency Department, Delta Hospital, Canada

DOI: 10.54026/CREM/1005

\section{Key Words}

Computerized Tomography; Clinical Symptoms; Emergency Department; Retropharyngeal Abscess; Sore Throat

Distributed under: Creative Commons CC-BY 4.0

\title{
Clinical Image: Sore Throat that cannot be Ignored
}

\author{
Jacky Sia ${ }^{*}$ \\ Emergency Department, Delta Hospital, Canada
}

\section{Abstract}

A 23-year-old man introduced to the emergency department three instances for one-week records of sore throat with a low-grade fever. Clinical signs and symptoms are non-specific, such as fever, sore throat, dysphagia, odynophagia comparable to acute pharyngitis. After one week of intravenous ceftriaxone and metronidazole, a repeated CT scan confirmed a recession of the abscess. Another study in Toronto revealed the sensitivity and specificity of CT. Complications, such as airway obstruction, mediastinitis, necrotizing fasciitis, carotid artery erosion, and sepsis can be fatal.

\section{Clinical Summary}

A 23-year man presented to the emergency department three times for one-week history of sore throat with a low-grade fever. On the first attendance, he was discharged with a presumptive diagnosis of acute pharyngitis. Despite several days of antibiotics, the pain did not show any improvement. X-ray of the neck was taken on his third attendance (Figure 1). Subsequent Computerized Tomography (CT) neck showed retropharyngeal abscess (Figure 2). After one week of intravenous ceftriaxone and metronidazole, a repeated CT scan showed a recession of the abscess (Figure 3). He was discharged with one week of oral antibiotic amoxicillin/clavulanate without surgical intervention.

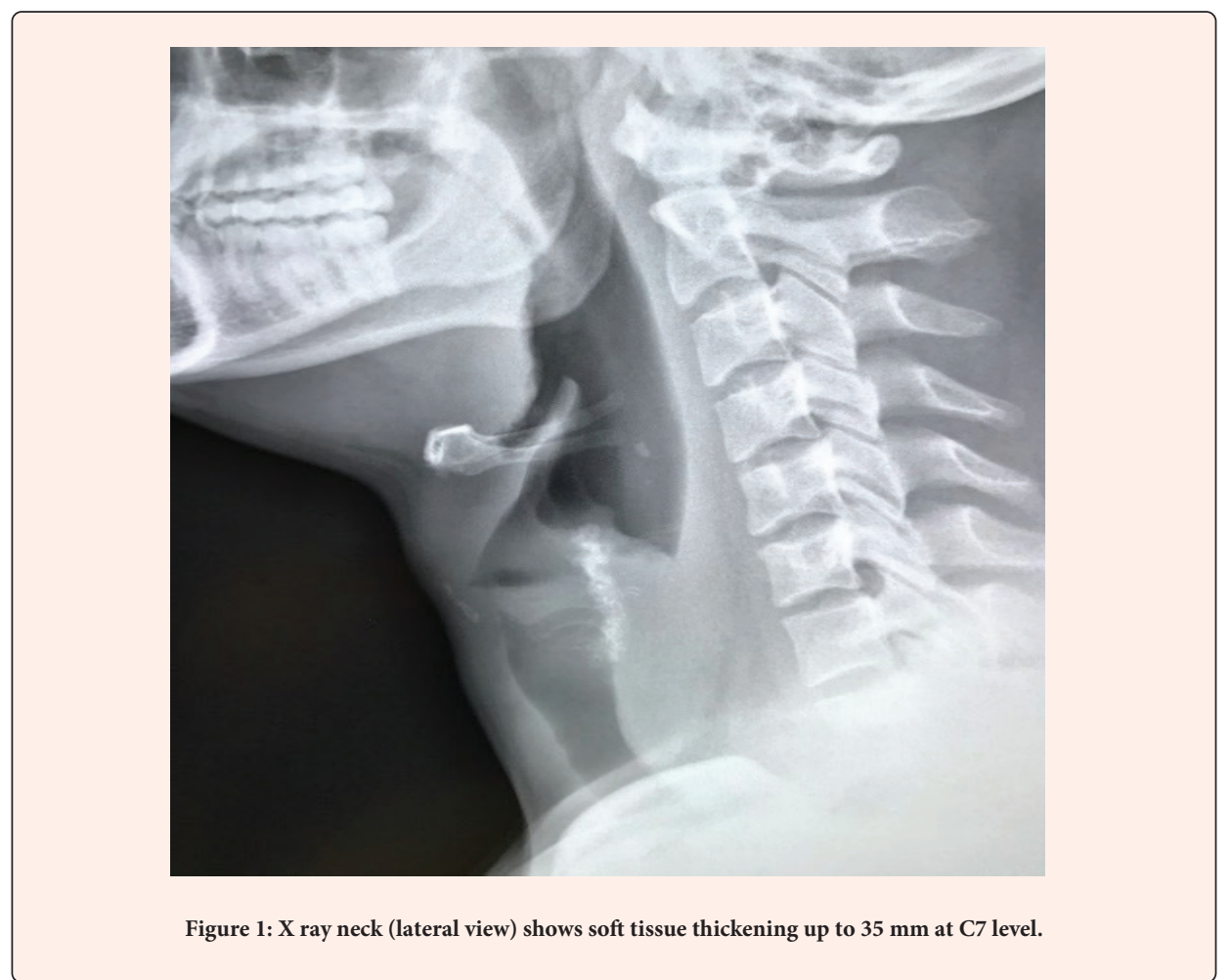

Retropharyngeal space is bounded by the skull base superiorly; constrictor muscles of the neck and its investing fascia anteriorly; the alar layer of the prevertebral fascia posteriorly; carotid sheath laterally and fascia at the level of C7 inferiorly. The space will regress after the age of six, and it's rare for an adult to have a non-traumatic retropharyngeal abscess.

Clinical symptoms are non-specific, including fever, sore throat, dysphagia, odynophagia similar to acute pharyngitis. Possible radiological features for RPA include the width of soft tissue greater than $50 \%$ of the width of the cervical vertebra at that level, straightening of cervical lordosis, non-traumatic subluxation of cervical vertebrae, gas or gas or gas fluid level in lateral neck X-ray. The sensitivity and specificity of lateral film X-ray were $80 \%$ and $100 \%$, respectively, whereas the sensitivity and specificity of computerized tomography of the neck were $100 \%$ and $45 \%$, respectively. Another study in Toronto revealed the sensitivity and specificity of CT were $81 \%$ and $57 \%$, respectively.

Early recognition is the key. Complications, such as airway obstruction, mediastinitis, necrotizing fasciitis, carotid artery erosion, and sepsis can be fatal. Management is a broad-spectrum intravenous antibiotic with or without surgical intervention. 


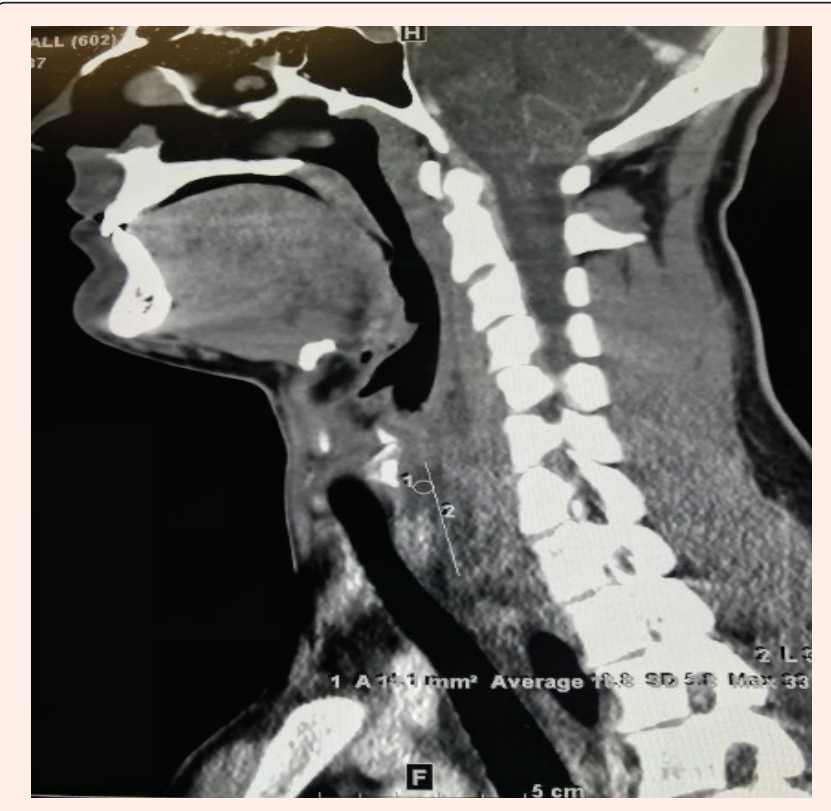

Figure 2: Computerized Tomography (CT) neck was performed showing 3.2 x 1.9 $\mathrm{cm} \times 7 \mathrm{~cm}$ Retro Pharyngeal Abscess (RPA) in craniocaudal length from C5 to T2, together with mediastinitis throughout the superior mediastinal fat. The glottis and trachea are displaced without compression.

Conflicts of Interest: None

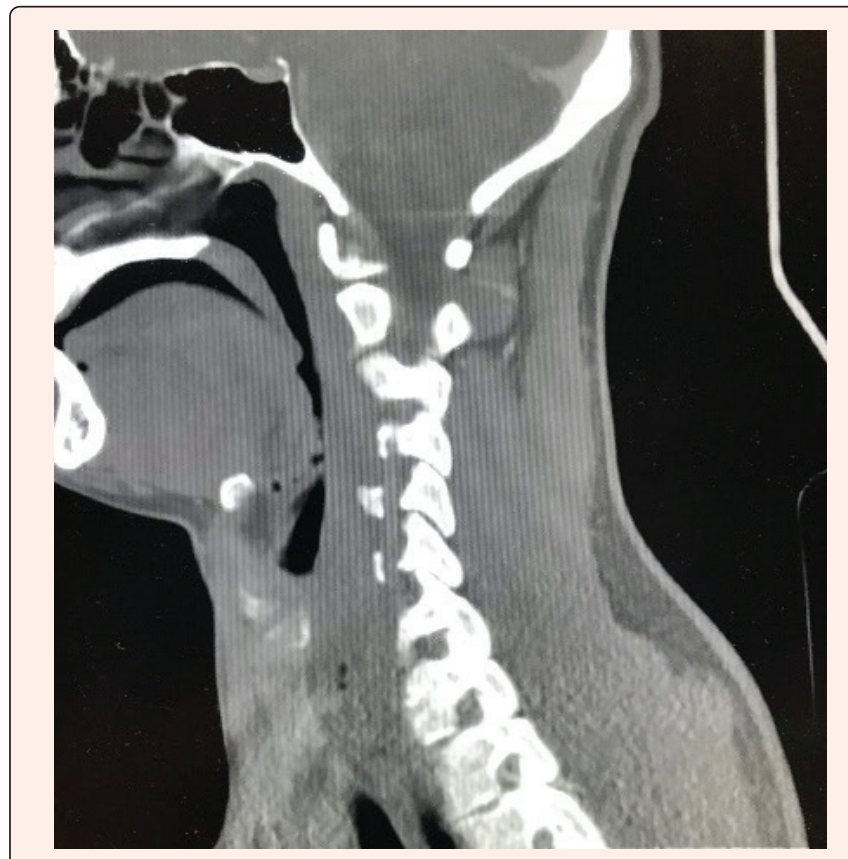

Figure 3: Recession of retropharyngeal abscess after intravenous antibiotics.

Financial Support: None 